\title{
Special issue on organic free radical chemistry
}

\author{
Mukund P. Sibi ${ }^{1 *}$, Chaozhong $\mathrm{Li}^{2^{*}} \&$ Ning Jiao ${ }^{3 *}$ \\ ${ }^{1}$ Department of Chemistry and Biochemistry, North Dakota State University, Fargo, ND, USA; \\ ${ }^{2}$ Shanghai Institute of Organic Chemistry, Chinese academy of Sciences, Shanghai 200032, China; \\ ${ }^{3}$ State Key Laboratory of Natural and Biomimetic Drugs, School of Pharmaceutical Sciences, Peking University, Beijing 100191, China
}

Received October 24, 2019; accepted October 24, 2019; published online October 30, 2019

Citation: Sibi MP, Li C, Jiao N. Special issue on organic free radical chemistry. Sci China Chem, 2019, 62: 1423-1424, https://doi.org/10.1007/s11426-0199643-y

The discipline of organic free radical chemistry dates back over 110 years since the discovery of triphenylmethyl radical by Moses Gomberg in 1900. Organic radical reactions became a thriving field in synthetic organic chemistry for a decade or so starting from the mid 1980s. Nevertheless, the significance of radicals in organic synthesis somehow remained hidden for a long period of time. The 1990s saw a renaissance of stereoselective radical reactions once deemed unattainable. The turn of the 20th century led to the development of new ways to carry out stereoselective radical reactions using organocatalysts. In the past decade, we have witnessed new directions for organic radical chemistry. It has now become an exciting area of research in organic chemistry, and new discoveries can be seen every day in various aspects of radical chemistry such as photoredox catalysis, transition-metal-catalyzed radical reactions, and application of electrochemistry. Besides the advances in new methodologies, the past decades have led to the development of a large tool box of reagents and catalysts to carry out radical reactions efficiently and selectively with an eye to meet the 21 st century metrics of low toxicity, minimal waste, green solvents, and sustainability.

The unique characteristics of radical reactions continue to be exploited by scientists to establish $\mathrm{C}-\mathrm{C}$ and $\mathrm{C}-\mathrm{X}$ bonds. These include installation of hindered centers, the ability to carry out cascade reactions, and multicomponent reactions to name a few.

This special issue collects 16 research articles and reviews, providing a glimpse of the state-of-the-art of free radical chemistry. They cover recent advances in various aspects of radical chemistry such as electrochemical reactions, photoredox or transition metal catalyzed radical reactions, radical cascades. It is very evident from the articles in this special issue that radical chemistry has a bright future and it will be a 'go to' area for solving important problems in synthetic organic chemistry.

*Corresponding authors (email: Mukund.Sibi@ndsu.edu; clig@mail.sioc.ac.cn; jiaoning@bjmu.edu.cn) 

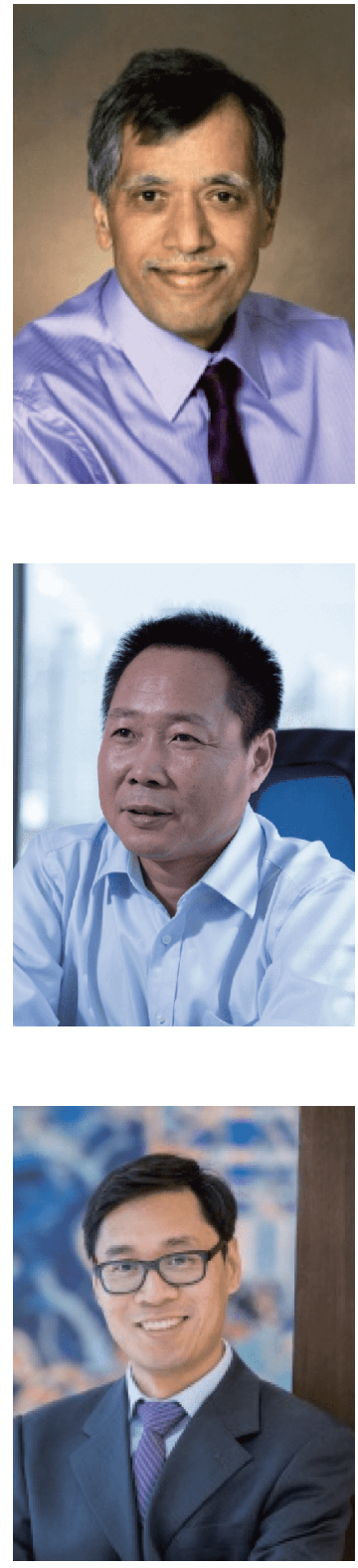

Mukund Sibi earned his PhD from the City University of New York. After postdoctoral studies, he began his independent career at North Dakota State University where he is currently a University Distinguished Professor. His research focus is on radical chemistry, development of enantioselective reactions, total synthesis, and green chemistry.

Chaozhong Li received his BSc degree from University of Science and Technology of China in 1988 and $\mathrm{PhD}$ degree from Shanghai Institute of Organic Chemistry (SIOC), Chinese Academy of Sciences (CAS) in 1993. After about 5 years' postdoctoral experience at Iowa State University, he returned to SIOC in 1999 and was promoted to Full Professor in 2000. His research interests focus on radicals in organic synthesis.

Ning Jiao received his $\mathrm{PhD}$ degree with Prof. Shengming Ma at Shanghai Institute of Organic Chemistry (SIOC) in 2004. He then spent 2004-2006 as an Alexander von Humboldt Postdoctoral Fellow with Prof. Manfred T. Reetz at Max Planck Institute für Kohlenforschung. In 2007, he joined the faculty at Peking University as an Associate Professor, and was promoted to Full Professor in 2010. His current research efforts are focused on: (1) aerobic oxidation, oxygenation, nitrogenation, and halogenation reactions; (2) the first-row transition metal catalysis and the inert chemical bonds activation. 